\title{
AN INEQUALITY FOR HARMONIC MAPS OF COMPACT KÄHLER MANIFOLDS THAT IMPLIES HOLOMORPHICITY
}

\author{
JAMES F. GLAZEBROOK
}

(Communicated by Jonathan M. Rosenberg)

\begin{abstract}
For harmonic maps of equidimensional compact Kähler manifolds satisfying certain conditions, a Chern class inequality is stated. If the map satisfies this inequality, it is holomorphic. The main result may be compared with a theorem of Eells and Wood for compact Riemann surfaces.
\end{abstract}

\section{INTRODUCTION}

Let $M$ and $N$ be compact Riemann surfaces with respective Euler characteristics $\chi(M)$ and $\chi(N)$. In [4], Eells and Wood consider harmonic maps $f: M \rightarrow N$ of degree $d_{f}$ and prove the following:

Theorem [4]. If $f: M \rightarrow N$ is a harmonic map relative to Riemannian metrics $g$ and $h$ on $M, N$ respectively, and if $\chi(M)+\left|d_{f} \chi(N)\right|>0$, then $f$ is \pm holomorphic relative to the complex structures determined by $g$ and $h$.

Here \pm holomorphic means holomorphic or antiholomorphic. Recently K. Ono [11] has proved an analogous result for harmonic maps from a compact Kähler manifold $M$ with first Chern class $c_{1}(M)<0$, to a compact Riemann surface $N$ of genus $\geq 2$.

The purpose of this paper is to state an analogue of the above theorem in higher dimensions, where $M$ and $N$ are both compact Kähler manifolds of equal (complex) dimension and where $N$ and the map satisfy certain hypotheses. Cases where these conditions are met are also discussed.

\section{THE HARMONICITY EQUATION}

Throughout, $M$ and $N$ will be taken to be compact Kähler manifolds. Let $f: M \rightarrow N$ be a smooth map. The complexified differential

$$
d^{\mathrm{C}} f: T^{\mathrm{C}} M \rightarrow f^{*} T^{\mathrm{C}} N
$$

Received by the editors November 3, 1988 and, in revised form, December 16, 1988.

1980 Mathematics Subject Classification (1985 Revision). Primary 58E20; Secondary 53C55.

Key words and phrases. Harmonic maps, pluriharmonic maps, holomorphic maps, Kähler manifolds, strongly seminegative curvature, Chern class, Kodaira surface. 
has a partial splitting in terms of complex types $(1,0)$ and $(0,1)$ :

$$
\begin{cases}\partial f: T^{1,0} M \rightarrow f^{*} T^{1,0} N, & \bar{\partial} f: T^{0,1} M \rightarrow f^{*} T^{1,0} N \\ \partial \bar{f}: T^{1,0} M \rightarrow f^{*} T^{0,1} N, & \overline{\partial f}: T^{0,1} M \rightarrow f^{*} T^{0,1} N .\end{cases}
$$

We note that $f$ is + holomorphic ( - holomorphic) if and only if $\partial \bar{f} \equiv 0$ (respectively, $\partial f \equiv 0)$. In what follows we shall let $E=\operatorname{Hom}\left(T^{1,0} M, f^{*} T^{1,0} N\right)$ and $F=\operatorname{Hom}\left(T^{1,0} M, f^{*} T^{0,1} N\right)$.

Let $\left\{z^{\alpha}\right\}$ and $\left\{\omega^{a}\right\}$ be local holomorphic coordinates on $M$ and $N$ respectively. Then on taking subscripts to denote partial derivatives, the local dictionary reads

$$
\begin{aligned}
\omega_{\alpha}^{a}(\leftrightarrow \partial f), \quad \bar{\omega}_{\alpha}^{a}(\leftrightarrow \partial \bar{f}), & \omega_{\bar{\alpha}}^{a}(\leftrightarrow \bar{\partial} f), \quad \bar{\omega}_{\bar{\alpha}}^{a}(\leftrightarrow \overline{\partial f}) \\
1 \leq \alpha \leq \operatorname{dim}_{\mathbf{C}} M, & 1 \leq a \leq \operatorname{dim}_{\mathbf{C}} N
\end{aligned}
$$

and the Kähler metrics of $M$ and $N$ are taken to be $g_{\alpha \bar{\beta}} d z^{\alpha} d \bar{z}^{\beta}$ and $h_{a \bar{b}} d \omega^{a} d \bar{\omega}^{b}$ respectively. If $f$ is harmonic, then the harmonicity equation is

$$
g^{\alpha \bar{\beta}} \omega_{\alpha \mid \bar{\beta}}=0
$$

where $\omega_{\alpha \mid \bar{\beta}}^{a}=\omega_{\alpha \bar{\beta}}^{a}+{ }^{N} \Gamma_{b c}^{a} \omega_{\alpha}^{b} \omega_{\bar{\beta}}^{c}$. We may also write

$$
\omega_{\alpha \mid \bar{\beta}}^{a}=D_{E}^{\prime \prime} \partial f
$$

where $D_{E}^{\prime \prime}$ is taken to denote covariant differentiation with respect to the $(0,1)$ part of the connection on the complex vector bundle $E$. As a consequence of the harmonicity of $f$, we have [13]

$$
0=h_{a \bar{b}} \omega_{\alpha \mid \bar{\gamma}}^{a} \bar{\omega}_{\beta \mid \mu}^{b} g^{\alpha \bar{\mu}} g^{\beta \bar{\gamma}}+R_{\bar{a} b \bar{c} d}^{N} \bar{\omega}_{\beta}^{a} \omega_{\alpha}^{b}\left(\bar{\omega}_{\bar{\mu}}^{c} \omega_{\bar{\gamma}}^{d}-\bar{\omega}_{\bar{\gamma}}^{c} \omega_{\bar{\mu}}^{d}\right) g^{\alpha \bar{\mu}} g^{\beta \bar{\gamma}}
$$

where $R_{\bar{a} b \bar{c} d}^{N}$ are the components of the curvature tensor of $N$. The above harmonicity equation can also be expressed as

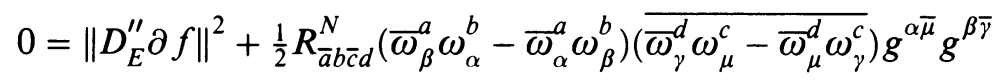

via a straightforward transformation in the second term. A similar expression holds when one replaces $E$ by $F$ and $\partial f$ by $\partial \bar{f}$.

\section{The MAIN Results}

Before exploiting the nature of equation (1.4), we shall state two general results which may be compared with the theorem of Eells and Wood [4] as stated in the Introduction. In what follows we shall assume $\operatorname{dim}_{\mathbf{C}} M=\operatorname{dim}_{\mathrm{C}} N=n$ and take $\omega$ to denote the Kähler class of $M$.

Theorem 2.1. Let $f: M \rightarrow N$ be a harmonic map. Assume that the following conditions are satisfied:

(i) the bundles $f^{*} T^{1,0} N$ and $f^{*} T^{0,1} N$ (hence $E$ and $F$ ) each have a holomorphic structure; 
(ii) $\partial f$ and $\partial \bar{f}$ are holomorphic sections of $E$ and $F$ respectively and at least one of these has maximal rank at some point;

(iii) the inequality

$$
c_{1}(M) \cdot \omega^{n-1}[M]+\left|f^{*} c_{1}(N) \cdot \omega^{n-1}[M]\right|>0
$$

is satisfied. Then $f$ is \pm holomorphic.

Proof. Taking the $n$th exterior product maps of $\partial f$ and $\partial \bar{f}$ in (1.1), we have

$$
\begin{aligned}
& \bigwedge^{n} \partial f: \bigwedge^{n} T^{1,0} M \rightarrow \bigwedge^{n} f^{*} T^{1,0} N \\
& \bigwedge^{n} \partial \bar{f}: \bigwedge^{n} T^{1,0} M \rightarrow \bigwedge^{n} f^{*} T^{0,1} N .
\end{aligned}
$$

Let $L_{f}$ and $L_{\bar{f}}$ denote the complex line bundles

$$
\begin{aligned}
& \left(\bigwedge^{n} T^{1,0} M\right)^{*} \otimes \bigwedge^{n} f^{*} T^{1,0} N \\
& \left(\bigwedge^{n} T^{1,0} M\right)^{*} \otimes \bigwedge^{n} f^{*} T^{0,1} N
\end{aligned}
$$

respectively. On taking first Chern classes, we have

$$
\begin{aligned}
& c_{1}\left(L_{f}\right)=-c_{1}(M)+f^{*} c_{1}(N) \\
& c_{1}\left(L_{\bar{f}}\right)=-c_{1}(M)-f^{*} c_{1}(N)
\end{aligned}
$$

(noting that $c_{1}\left(\left(\bigwedge^{n} T^{1,0} M\right)^{*}\right)=c_{1}\left(K_{M}\right)=-c_{1}(M)$, where $K_{M}$ denotes the canonical line bundle of $M$, etc.).

On account of conditions (i) and (ii) and the fact that holomorphicity is preserved under the operation of exterior product, we deduce that $\Lambda^{n} \partial f$ and $\bigwedge^{n} \partial \bar{f}$ are holomorphic sections of the holomorphic line bundles $L_{f}$ and $L_{\bar{f}}$ respectively. Thus the first Chern classes regarded as effective divisors on $M$ [6], have non-negative intersection with the $(n-1)$ th power of the (positive) Kähler class $\omega$ of $M$ and we obtain

$$
c_{1}\left(L_{f}\right) \cdot \omega^{n-1}[M]=-c_{1}(M) \cdot \omega^{n-1}[M]+f^{*} c_{1}(N) \cdot \omega^{n-1}[M] \geq 0
$$

and

$$
c_{1}\left(L_{\bar{f}}\right) \cdot \omega^{n-1}[M]=-c_{1}(M) \cdot \omega^{n-1}[M]-f^{*} c_{1}(N) \cdot \omega^{n-1}[M] \geq 0 .
$$

Now let us suppose that $f$ is non \pm holomorphic in a neighborhood of a point where at least one of $\partial f, \partial \bar{f}$ is of maximal rank. Then at least one of the holomorphic sections $\Lambda^{n} \partial f, \Lambda^{n} \partial \bar{f}$ is non-zero there. Thus at least one of (2.6), (2.7) is $>0$. But the inequality (2.1) as stated in the theorem implies that one of these would be negative and hence a contradiction is reached. Therefore $f$ is \pm holomorphic in a neighborhood of this point and hence throughout $M$ by [14, Proposition 4].

Remarks. 1. If $M$ has constant scalar curvature $R_{s}$, then $c_{1}(M) \cdot \omega^{n-1}[M]=$ $R_{s} / \pi n\left(\omega^{n} \cdot[M]\right)$.

2. In terms of the canonical divisors $K_{M}$ and $K_{N}$ of $M$ and $N$, respectively, if $K_{M}$ and $K_{N}$ are both $>0$, then the inequality (2.1) is satisfied for $f^{*} K_{N}>K_{M}$ as is easily deduced. 
In the next section we shall exemplify some cases where the hypotheses of the above theorem are satisfied. For the moment, we see that an inspection of the details of the proof of Theorem 2.1 allows an interpretation in terms of analytic sets and the zeros of $\Lambda^{n} \partial f, \Lambda^{n} \partial \bar{f}$; this in fact closely parallels the arguments in [4] when applied to higher dimensions.

To see this, let $M_{1}$ be an analytic set of (complex) codimension 1 in $M$. Then formally, $M_{1}=\sum_{i} M_{1, i}$ where $M_{1, i}$ denotes an irreducible component of $M_{1}$, and this expresses $M_{1}$ as an effective divisor on $M$ in a natural way. Let $s$ be a smooth section of a complex vector bundle $L \rightarrow M$. Then $s$ is locally definable by a smooth function $g$ and it is assumed that $g$ vanishes on a neighborhood of $0 \in \mathbf{C}^{n}$ along an irreducible component of $M_{1}$, i.e., $g\left(z^{1}, \ldots, z^{n-1}, z^{n}\right)=0$ along $\left\{z^{n}=0\right\}$. For any fixed $\left(z^{1}, \ldots, z^{n-1}\right)$, we define for $z^{n} \in \mathbf{C}$, the map $\hat{g}=g /|g|: S^{1} \rightarrow S^{1}$. We say that $s$ vanishes to order $r$ on this component, if degree $\hat{g}=r$. If $L$ is a line bundle, it follows that $c_{1}(L)=\sum_{i} n_{i} M_{1, i}$, where $n_{i}$ denotes the order of vanishing of $s$ along $M_{1, i}$. The proof of Theorem 2.2 was based on the fact that $c_{1}\left(L_{f}\right) \cdot \omega^{n-1}[M]$ and $c_{1}\left(L_{\bar{f}}\right) \cdot \omega^{n-1}[M]$ were both $\geq 0$. The reasoning behind the proof may be applied to establish:

Theorem 2.2. Let $f: M \rightarrow N$ be a harmonic map and assume that

(i) the sections $\bigwedge^{n} \partial f, \bigwedge^{n} \partial \bar{f}$ vanish to respective orders $m_{i}, n_{i} \geq 0$ along the irreducible components $M_{1, i}$ of an analytic set $M_{1}$ in $M$, $\operatorname{codim}_{\mathbf{C}} M_{1}=1$ where in the set $\left\{m_{i}, n_{i}\right\}$ not all elements are zero;

(ii) the inequality (2.1) is satisfied. Then $f$ is \pm holomorphic.

Proof. Suppose $f$ is harmonic, non \pm holomorphic in some open set along $M_{1}$. Condition (i) implies that

$$
\begin{aligned}
& c_{1}\left(L_{f}\right) \cdot \omega^{n-1}[M]=\left(\sum_{i} m_{i} M_{1, i}\right) \cdot \omega^{n-1}[M] \geq 0 \\
& c_{1}\left(L_{\bar{f}}\right) \cdot \omega^{n-1}[M]=\left(\sum_{i} n_{i} M_{1, i}\right) \cdot \omega^{n-1}[M] \geq 0
\end{aligned}
$$

with at least one of these positive. Again, inequality (2.1) gives rise to a contradiction and the result follows as in the proof of Theorem 2.1.

Thus we see that a general harmonic map, even with a semblance of complex analyticity as suggested by hypothesis (i) of the above theorem (cf [7]), is still some way from being \pm holomorphic, thus confirming the very rigid nature of the latter.

\section{SOME EXAMPLES}

In this section we shall study some cases where the hypotheses (i) and (ii) of Theorem 2.1 are satisfied. For the following discussion, we do not need equality 
of dimension. What figures prominently here, is the nature of (1.4):

$$
0=\left\|D_{E}^{\prime \prime} \partial f\right\|^{2}+\frac{1}{2} R_{\bar{a} b \bar{c} d}^{N}\left(\bar{w}_{\beta}^{a} w_{\alpha}^{b}-\bar{w}_{\alpha}^{a} w_{\beta}^{b}\right)\left(\overline{\bar{w}_{\gamma}^{d} w_{\mu}^{c}-\bar{w}_{\mu}^{d} w_{\gamma}^{c}}\right) g^{\alpha \bar{\mu}} g^{\beta \bar{\gamma}} .
$$

Firstly, following [14][13], we say that $N$ has strongly seminegative curvature if it is assumed that the second term on the right-hand side of (1.4) is $\geq 0$. But then, so too is the first term and hence both terms in (1.4) must vanish. Secondly, a map $f: M \rightarrow N$ is said to be pluriharmonic (or $(1,1)$-geodesic) if

$$
(\nabla d f)^{(1,1)}=0
$$

i.e., the $(1,1)$ component of the (complexified) second fundamental form of $f$ vanishes. This condition is stronger than harmonicity of $f$ but weaker than holomorphicity. Such a map satisfies

$$
D_{E}^{\prime \prime} \partial f=0 \quad \text { and } \quad D_{F}^{\prime \prime} \partial \bar{f}=0
$$

(see e.g. $[17, \S 1])$. Thus if $N$ has strongly seminegative curvature, then any harmonic map $f: M \rightarrow N$ is pluriharmonic.

Now let us suppose that $N$ is one of the following:

(i) $N$ is a Hermitian symmetric space of compact type, or

(ii) $N$ has a universal covering which is a locally Hermitian symmetric space of non-compact type.

Regarding $N$ as a Riemannian locally symmetric space, we let $\langle$,$\rangle and R^{N}$ denote the complex multilinearity extension to $T^{\mathrm{C}} N$ of the Riemannian metric and curvature tensor of $N$ respectively, as in $\S 1$. If $f: M \rightarrow N$ is harmonic, then following [3][13], (1.4) can be restated as the following: for $x \in M$,

$$
0=\left\|D^{0,1} d^{\prime} f\right\|^{2}-\left\langle R^{N}(X, Y) \bar{X}, \bar{Y}\right\rangle \quad X, Y \in d_{x}^{\prime} f\left(T^{1,0} M\right)
$$

where $d^{\prime} f=\partial f+\partial \bar{f}$. If $f$ is taken to be pluriharmonic, then (3.1) implies the vanishing of the first term in (3.2) and hence the second term automatically vanishes. Thus

$$
0=\left\langle R^{N}(X, Y) \bar{X}, \bar{Y}\right\rangle=\langle[X, Y],[\bar{X}, \bar{Y}]\rangle .
$$

We shall also consider the case

(iii) $N$ is covered by the $n$-polydisc.

In cases (ii) and (iii) above, $N$ has the property of strongly seminegative curvature. Note that in (i) and (ii), the sign of the curvature term differs only by that of the corresponding Killing form. These observations lead to the following:

Proposition 3.1 (cf [3], [15], [16]). Let $M, N$ have arbitrary dimensions and let $f: M \rightarrow N$ be harmonic. Assume that one of the following holds:
(a) case (i) above and $f$ is pluriharmonic;
(b) case (ii) above;
(c) case (iii) above. 
Then the complex vector bundles $f^{*} T^{1,0} N$ and $f^{*} T^{0,1} N$, hence $E$ and $F$, each have a natural holomorphic structure and $\partial f$ and $\partial \bar{f}$ are holomorphic sections of $E$ and $F$ respectively.

Proof. We firstly consider (a) and (b), noting that in case (b), $N$ has strongly seminegative curvature and hence (3.1) holds. Thus pluriharmonicity is automatic in case (b).

From (3.3), following [3, Theorem (2.2)] [13], we see that $d^{\mathrm{C}} f$ maps $T^{1,0} M$ onto an abelian subspace of $T^{\mathrm{C}} N$, i.e.,

$$
\left[d^{\mathbf{C}} f(Z), d^{\mathbf{C}} f(W)\right]=0 \quad \text { for all } Z, W \in T^{1,0} M \text {. }
$$

Let

$$
\begin{aligned}
& d^{1,0} f: T^{\mathrm{C}} M \rightarrow f^{*} T^{1,0} N \\
& d^{0,1} f: T^{\mathbf{C}} M \rightarrow f^{*} T^{0,1} N
\end{aligned}
$$

be defined by $d^{1,0} f=\partial f+\bar{\partial} f$ and $d^{0,1} f=\partial \bar{f}+\overline{\partial f}$. As in [3, Theorem (2.3)], the integrability condition for a holomorphic structure on $f^{*} T^{1,0} N$ is equivalent to

$$
R^{N}\left(d^{1,0} f(X), d^{1,0} f(Y)\right)=0 \quad \text { for } X, Y \in T^{0,1} M
$$

regarding $d^{1,0} f \in \mathscr{C}\left(f^{*} T^{1,0} N\right)$ by abuse of notation. Now $R^{N}\left(d^{1,0} f(X)\right.$, $\left.d^{1,0} f(Y)\right)=0$ is equivalent to $\left[d^{1,0} f(X), d^{1,0} f(Y)\right]=0$. But the latter is equivalent to $\left[d^{0,1} f(\bar{X}), d^{0,1} f(\bar{Y})\right]=0$ since the Lie bracket is a real operator and the vanishing results from the harmonicity of $f$. Thus $f^{*} T^{1,0} N$ has a holomorphic structure and likewise $f^{*} T^{0,1} N$. The corresponding result for the $n$-polydisc case in (c), was proved in [15] (see also [16]). It then follows that $E$ and $F$ each have a natural holomorphic structure. The conditions in (3.1) resulting from the pluriharmonicity in (a) and the strongly seminegative curvature condition in (b) and (c), imply the last assertion.

Remark. Recall that a harmonic map is stable if the second variation of the energy

$$
E(f)=\frac{1}{2} \int_{M}|d f|^{2} \nu_{M}
$$

is non-negative. When $M=\mathbf{C} P^{n}$ and $N$ is an irreducible Hermitian symmetric space of compact type, a stable harmonic map into $N$ is known to be pluriharmonic [2] [10] and, in particular, holomorphic [2]. It is straightforward to see that (2.1) is satisfied in this case (cf. the first remark in §2). For $n=2$, when $M$ is a compact Kähler surface and $N$ is of the above type, the holomorphicity of stable harmonic maps was discussed in [1] and [2].

Example. Following the second remark in $\S 2$, we wish to consider cases where the condition $f^{*} K_{N}>K_{M}$ is satisfied.

Some possible sources of examples outside of Siu's rigidity criterion (see [15][16]) are provided by the following. Firstly, both $M$ and $N$ may 
be taken to be covered by the $n$-polydisc. Secondly, for $n=2$, we may take $M$ to be a Kodaira surface, generally exhibited in terms of a ramified covering map $M \rightarrow N$ where $N=P \times Q$ is a product of compact Riemann surfaces $P$ and $Q$, each of genus $\geq 2$, [9] (see also [8]). Both $K_{M}$ and $K_{N}$ are known to be ample in this case and thus $K_{M}, K_{N}>0$. The only known examples are those whereby the map is a holomorphic ramified covering, and hence $\partial f$ is of maximal rank outside of an analytic curve in $M$. However, we may use this to formulate a short recipe for realizing the above inequality. Choose complex structures $J_{1}$ and $J_{2}$ such that

(i) $J_{1}=\bar{J}_{M}$ the conjugate complex structure of $M\left(K_{M} \rightarrow \bar{K}_{M}\right)$.

(ii) $J_{2}$ is chosen, such that $f:\left(M, J_{1}\right) \rightarrow\left(N, J_{2}\right)$ is - holomorphic. Then by the - holomorphic Riemann-Hurwitz formula (2.5) applied to (i) and (ii), we have

$$
f^{*} \bar{K}_{N}<\bar{K}_{M}
$$

which is the desired condition (noting $\bar{K}_{M}=K_{M}^{*}$, etc.), and likewise $\partial \bar{f}$ has maximal rank off an analytic curve in $M$. Now deform $J_{1}$ and $J_{2}$ by a sufficiently small amount such that (3.4) holds; this is always possible, without changing the rank condition. The deformation does not, a priori, imply that $f$ remains - holomorphic or becomes + holomorphic. Now $N$ has strongly seminegative curvature and the theorem of Eells-Sampson [5] implies that a harmonic representative exists in a given homotopy class of such maps. However, on account of Theorem 2.1, (3.4) implies that $f$ does indeed remain in the holomorphic category under this deformation. One might also consider the above construction applied to an arbitrary $k$-fold product of such maps $f=\left(f_{1} \times \cdots \times f_{k}\right): M_{1} \times \cdots \times M_{k} \rightarrow\left(P_{1} \times Q_{1}\right) \times \cdots \times\left(P_{k} \times Q_{k}\right) \quad(n=2 k)$ where each $M_{i}, 1 \leq i \leq k$, is a Kodaira surface and $P_{i}, Q_{i}$ are as above. Taking $M=M_{1} \times \cdots \times M_{k}, N=\left(P_{1} \times Q_{1}\right) \times \cdots \times\left(P_{k} \times Q_{k}\right), M$ and $N$ may be endowed with complex structures which are not, a priori, the appropriate product structures that would imply $f$ is \pm holomorphic.

Taking account of the above results which we have established, we arrive at the following two theorems:

Theorem 3.2. Let $\operatorname{dim}_{\mathrm{C}} M=\operatorname{dim}_{\mathrm{C}} N=n$ and assume that $N$ is either

(i) covered by a locally Hermitian symmetric space of non-compact type, or

(ii) covered by the n-polydisc.

Then any harmonic map $f: M \rightarrow N$ having at least one of $\partial f, \partial \bar{f}$ of maximal rank at some point satisfies

$$
\left|f^{*} c_{1}(N) \cdot \omega^{n-1}[M]\right| \geq c_{1}(M) \cdot \omega^{n-1}[M] .
$$


Proof. If the contrary to the inequality (3.5) is assumed, then

$$
0 \leq\left|f^{*} c_{1}(N) \cdot \omega^{n-1}[M]\right|<c_{1}(M) \cdot \omega^{n-1}[M],
$$

would imply that $f$ is \pm holomorphic by (2.1). In which case, on combining (2.6) and (2.7), we have

$$
\left|f^{*} c_{1}(N) \cdot \omega^{n-1}[M]\right| \geq c_{1}(M) \cdot \omega^{n-1}[M],
$$

a contradiction.

In a similar vein, we have the following theorem, whose proof by contradiction follows that of Theorem 3.2.

Theorem 3.3. Let $\operatorname{dim}_{\mathrm{C}} M=\operatorname{dim}_{\mathrm{C}} N=n$ and assume that $N$ is an Hermitian symmetric space of compact type. Then any pluriharmonic map $f: M \rightarrow N$ having at least one of $\partial f, \partial \vec{f}$ of maximal rank at some point, satisfies (3.5).

We remark that this last inequality may be compared with the general restrictions on equidimensional holomorphic maps, without the assumption on $N$. For instance, compare with the following: $\mathbf{C} P^{2}$ could never be exhibited as a holomorphic ramified covering of a $K-3$ surface $\left(c_{1}(N)=0\right)$. In fact, if $\mathbf{C} P^{n} \rightarrow N$ is such a map, then it is well known that $N \simeq \mathbf{C} P^{n}$. More generally, any $n$-dimensional algebraic manifold can be exhibited as such a ramified covering of $\mathbf{C} P^{n}$ (this is also well known, see e.g. [12]).

\section{ACKNOWLEDGMENT}

The author wishes to thank D. Burns, R. Simha and D. Toledo for interesting discussions and The Institute For Advanced Study for its hospitality.

\section{REFERENCES}

1. D. Burns and P. de Bartolomeis, Applications harmoniques stables dans $\mathbf{P}^{n}$, Ann. Sci. École Norm. Sup (4) 21 (1988), no. 2, 159-177.

2. D. Burns, F. Burstall, P. de Bartolomeis and J. Rawnsley, Stability of harmonic maps of Kähler manifolds, preprint, University of Warwick, 1978.

3. J. A. Carlson and D. Toledo, Harmonic mappings of Kähler manifolds to locally symmetric spaces, preprint, Publ. Math. I.H.E.S. (to appear).

4. J. Eells and J. C. Wood, Restrictions on harmonic maps of surfaces, Topology 15 (1976), 263-266.

5. J. Eells and J. H. Sampson, Harmonic mappings of Riemannian manifolds, Amer. J. Math. 86 (1964), 109-160.

6. P. A. Griffiths and J. Harris, Principles of algebraic geometry, Wiley Interscience, 1978.

7. R. C. Gunning, Lectures on complex analytic varieties: the local parametrization theorem, Princeton University Press, 1970.

8. J. Jost and S. T. Yau, Harmonic mappings and Kähler manifolds, Math. Ann. 262 (1983), 145-166.

9. K. Kodaira, A certain type of irregular algebraic surfaces, J. Anal. Math. 19 (1967), 207-215.

10. Y. Ohnita and S. Udagawa, Stable harmonic maps from Riemann surfaces to compact Hermitian symmetric spaces, Tokyo J. Math. Vol. 10, No. 2 (1987), 385-390. 
11. K. Ono, On the holomorphicity of harmonic maps from compact Kähler manifolds to hyperbolic Riemann surfaces, Proc. Amer. Math. Soc., vol. 102, no. 4, (1988), 1071-1076.

12. R. Remmert and T. Van de Ven, Über holomorphe Abbildungen projectivalgebraicher Mannigfaltigkeiten auf komplexe Räume, Math. Ann. 142 (1961), 453-486.

13. J. H. Sampson, Applications of harmonic maps to Kähler geometry, Contemp. Math. 49 (1986), 125-133.

14. Y. T. Siu, The complex analyticity of harmonic maps and the strong rigidity of compact Kähler manifolds, Ann. of Math. 122 (1980), 73-111.

15. __ Some recent results in complex manifold theory related to vanishing theorems for semipositive case, Springer Lecture Notes in Math. 1111 (1985), 169-192.

16. _ Strong rigidity for Kähler manifolds and the construction of bounded holomorphic functions, in Discrete Groups in Geometry and Analysis, Papers in Honor of G. D. Mostow on His Sixtieth Birthday, R. Howe (ed.), Birkhauser, Boston, 1987, 124-151.

17. S. Udagawa, Pluriharmonic maps and minimal immersions of Kähler manifolds, J. London Math. Soc. (2), 37 (1988), 375-384.

Department of Mathematics, EAstern Illinois University, Charleston, Illinois 61920 